\title{
A Multivariate Prognostic SCORE FOR PREDICTING MORTALITY OF ACQUIRED IMMUNODEFICIENCY SYNDROME PATIENTS WITH HYPOXEMIC RESPIRATORY FAILURE AND PNEUMOCYSTIS JIROVECI PNEUMONIA
}

\author{
Carmen M. Hernández-Cárdenas ${ }^{1}$, Gastón Mendoza-Copa ${ }^{1}$, Paola Hong-Zhu², \\ ItZel A. Gómez-García ${ }^{2}$ and Gustavo Lugo-Goytia ${ }^{1 *}$ \\ ${ }^{1}$ Respiratory Intensive Care Unit, Instituto Nacional de Enfermedades Respiratorias Ismael Cosío Villegas, \\ Mexico City, ${ }^{2}$ School of Medicine, Instituto Tecnológico de Monterrey, Campus Ciudad de México, Mexico City, \\ Mexico.
}

\begin{abstract}
Background: Severe hypoxemic respiratory failure (SHRF) due to Pneumocystis jiroveci pneumonia (PJP) in AIDS patients represents the main cause of admission and mortality in respiratory intensive care units (RICUs) in low- and middle-income countries. Objective: The objective of this study was to develop a predictive scoring system to estimate the risk of mortality in HIV/ AIDS patients with PJP and SHRF. Methods: We analyzed data of patients admitted to the RICU between January 2013 and January 2018 with a diagnosis of HIV infection and PJP. Multivariate logistic regression and Kaplan-Meier method were used in data analysis. The RICU and inhospital mortality were $25 \%$ and $26 \%$, respectively. Multivariate analysis identified four independent predictors: body mass index, albumin, time to ICU admission, and days of vasopressor support. A predictive scoring system was derived and validated internally. The discrimination was 0.869 ( $95 \%$ confidence interval: $0.821-0.917$ ) and calibration intercept $(\alpha)$ and slope $(\beta)$ were 0.03 and 0.99 , respectively. The sensitivity was $47.2 \%$, specificity was $84.6 \%$, positive predictive value was $89.2 \%$, and negative predictive value was $82.6 \%$. Conclusions: This scoring system is a potentially useful tool to assist clinicians, in low- and medium-income countries, in estimating the RICU and inhospital mortality risk in patients with HIV/ AIDS and SHRF caused by PJP. (REV INVEST CLIN. 2019;71:311-20)
\end{abstract}

Key words: Intensive care units. Severe hypoxemic respiratory failure. Pneumocystis jiroveci pneumonia. Human immunodeficiency virus. Acquired immunodeficiency syndrome. Prognostic factors. Mortality.

Corresponding author:

*Gustavo Lugo Goytia

Respiratory Intensive Care Unit

Instituto Nacional de Enfermedades Respiratorias

Ismael Cosío Villegas

Calzada de Tlalpan, 4502

Col. Belisario Domínguez Secc. 16, Del. Tlalpan

C.P. 14080, Ciudad de México, México

E-mail: lugogoy@yahoo.com.mx

Received for publication: 11-01-2019

Approved for publication: 11-04-2019

DOI: $10.24875 / R I C .19002957$ 


\section{INTRODUCTION}

HIV infection continues to be a serious health hazard, particularly in developing countries. In Mexico, in 2016, there were 200,000-240,000 people living with HIV, 11,000-14,000 new HIV infections, and 3300-5300 AIDS-related deaths ${ }^{1}$. A high percentage of HIV/AIDS patients requires intensive care $^{2}$, although their mortality rates have improved over the past decades ${ }^{2-6}$. In addition, the causes of admission into intensive care units (ICUs) have changed since the introduction of highly active antiretroviral therapy $(A R V T)^{7,8}$. In high-income countries, opportunistic respiratory infections such as Pneumocystis jiroveci pneumonia (PJP) no longer represent the primary reason for ICU admission in patients with HIV infection. However, in developing countries, a high percentage of individuals are unaware that they are carriers of HIV and have not received ARVT or prophylaxis ${ }^{9,10}$. These patients represent the greatest number of admissions to respiratory ICUs (RICUs) due to severe acute hypoxemic respiratory failure (SAHRF) secondary to PJP. Therefore, tools that allow the prediction of mortality are needed to assist in clinical decisionmaking, optimizing the limited health resources available, and communicating realistic expectations regarding prognosis to patients and their families. The objectives of this study were to describe the clinical characteristics and outcomes of a prospective cohort of patients with SAHRF, HIV infection, and PJP requiring RICU admission and determine the variables associated with mortality to develop a predictive scoring system for inhospital mortality.

\section{METHODS}

\section{Design and patients}

We conducted an observational study of a prospective cohort admitted to the RICU at the National Institute of Respiratory Diseases in Mexico City, Mexico. This is a 241-bed third-level teaching and research reference center for patients with HIV infection and respiratory diseases with a 15-bed RICU. All patients who were admitted to the RICU between January 2013 and January 2018 with a diagnosis of HIV infection (confirmed by serological tests and viral load) and SAHRF and PJP (confirmed by aspirate, BAL, and/or biopsy) that required invasive mechanical ventilation were included in the analysis. We excluded patients with evidence of coinfection due to bacteria or fungi on admission (documented by microbiological study of aspirate, BAL, and/or biopsy). If a patient was admitted to the RICU on more than one occasion, only the data from the first admission were considered. Due to the study design, the ethics committee did not request informed consent from the patients or their kin. The study was registered and approved by the institution's biomedical and ethics research committee and adhered to the TRIPOD guidelines on prediction model development and validation studies ${ }^{11}$.

\section{Variables and definitions}

The patients' clinical data were obtained from the electronic database and clinical records. The following data were collected by two researchers: demographic information; risk factors for HIV infection; previous diagnosis of HIV infection; treatment with ARV drugs and anti-infective prophylaxis; time between admission to the emergency room and transfer to the RICU; acute kidney injury defined according to the classification of Mehta et al. ${ }^{12}$; hemodynamic and respiratory parameters on RICU admission; markers of infection; anthropometric data comprising weight, height, and body mass index (BMI: weight/height ${ }^{2}$ ); and the sequential organ failure assessment (SOFA) score obtained in the first $24 \mathrm{~h}$ of admission to RICU ${ }^{13}$. PJP was diagnosed by tracheal aspiration or bronchoalveolar lavage, which was performed when analysis of the tracheal sample did not allow for identification of the microorganism. Samples were examined after applying the Gomori methenamine silver and Giemsa stains; moreover, all samples were evaluated for viruses, bacteria, and fungi. ARDS was defined according to the recent Berlin definition ${ }^{14}$. The primary endpoint was inhospital mortality, defined as death before discharge, regardless of the length of stay.

\section{Statistical analysis}

Data analysis was performed using the statistical software SPSS version 21.0 (IBM Statistics, Armonk, New York). Data were summarized as counts or percentages for categorical variables and the median with interquartile range $\left(25^{\text {th }}-75^{\text {th }}\right.$ percentiles) for continuous variables. Group comparisons were made by applying Chi-squared test for categorical variables and the Wilcoxon-Mann-Whitney test for continuous 
variables. All tests of significance were two-tailed, and $\mathrm{p}<0.05$ was considered to be statistically significant.

\section{Missing data and multiple imputation}

The amount of missing data was determined, and the candidate variables were excluded if more than $25 \%$ of the total data for that variable were unavailable. Before the imputations, a test was performed to determine the randomness of the lost data to avoid bias. The imputation of the missing candidate variables was performed using the linear regression method for continuous variables and logistic regression for the nominal variables. After five imputations, the average values of the observed data were compared with those of the imputed data; as there were no significant differences, the imputed database was used for the statistical analysis ${ }^{15,16}$.

\section{Logistic regression modeling}

The binary logistic regression analysis included the candidate variables selected as independent covariates and inhospital mortality as the dependent variable. The candidate variables were first tested for inclusion in univariate models; those that were significant based on $p<0.20$ were entered into multivariable models, and a series of progressive backward eliminations was used to identify the variables for the final model ${ }^{17}$. To ensure that the scoring system was suitable for use at the bedside, the number of variables was reduced to ensure simplicity and clinical relevance. The results are presented as adjusted odds ratios with $95 \%$ confidence intervals (Cls) and regression coefficients ( $\beta$ values).

\section{Conversion to the clinical prediction rule}

The logistic regression model was converted into a clinical prediction score by transforming the estimates of the individual parameters of the model into weights (risk units) according to the method used in the Framingham Heart Study ${ }^{18}$. A risk unit was represented by the regression coefficient associated with a BMI decrease of 1.5 units $\left(\mathrm{kg} / \mathrm{m}^{2}\right)$. The points were assigned to all other variables, where their regression coefficients were divided by the BMI coefficient. The individual risk of the patient was estimated by calculating the sum of the scores from the risk factors. A new logistic regression analysis was performed using the predictive score as the only independent variable and the inhospital mortality as the dependent variable. Low-, intermediate-, and high-risk strata were defined based on the estimated probability of mortality. We present the predicted inhospital mortality risks for the different scores together with the sensitivity, specificity, and inhospital mortality observed in the sample of complete derivation. The score was evaluated using the model's diagnosis ${ }^{19}$. The overall predictive performance of the score was evaluated using the NagelkerkeR ${ }^{2}$ for binary exposure ${ }^{20}$, which refers to the proportion of variation explained by the model. Discrimination, which refers to the ability of the scoring system to distinguish patients who die from those who are alive, was evaluated using the area below the receiver operating characteristic (ROC) curve ${ }^{21}$. Finally, the calibration was evaluated as recommended in the literature through calibration and intersection graphs of $\alpha$ and slope $\beta$ of the regression of the binary result (inhospital mortality) in the predicted values, complemented by the Hosmer-Lemeshow goodness-of-fit statistic ${ }^{22}$.

\section{Internal validation}

The model was validated internally using bootstrapping $^{23}$. One thousand random samples with replacement were obtained from the original cohort. The model was developed again in each bootstrap sample to yield new parameter values for each bootstrap model. Finally, the mean, standard error, and $\mathrm{Cls}$ of the coefficients were obtained.

\section{Sample size and power}

A power analysis indicated that a logistic regression model with 202 patients and 54 events would have sufficient power to include four predictive variables ${ }^{24}$.

\section{RESULTS}

\section{Demographic and clinical characteristics of patients}

During the study period, 1415 admissions to the RICU were registered, of which 1235 were non-HIVrelated and were excluded. The diagnosis of HIV infection was confirmed in 219 patients, 17 of whom 
Figure 1. Flow diagram of HIV/AIDS patients enrolled in the study at RICU from January 2013 to January 2018.

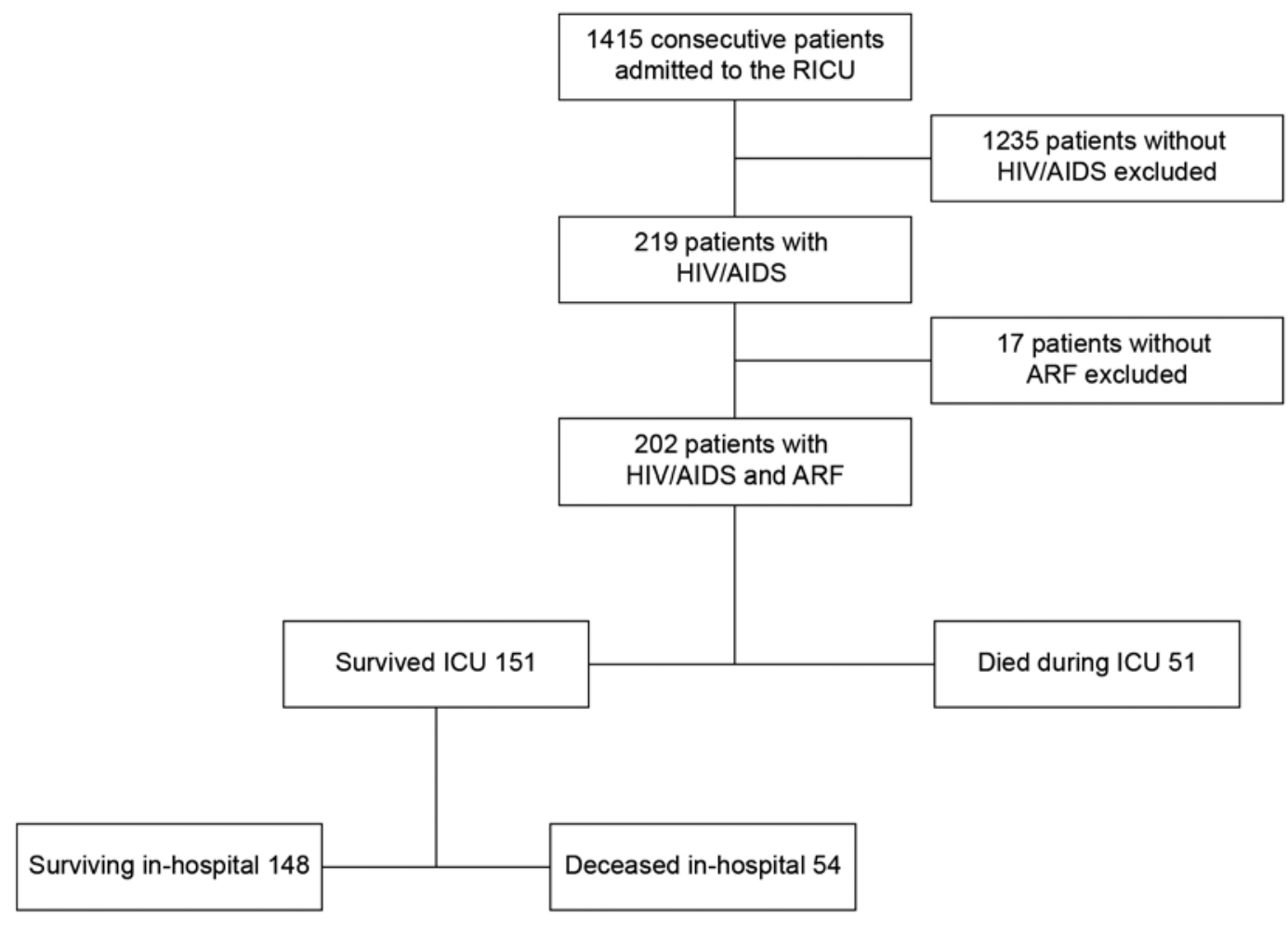

were excluded because they did not meet the inclusion criteria. Therefore, the final analyzed sample comprised 202 patients (Fig. 1).

The general characteristics of surviving and non-surviving patients are shown in Table 1 . The age and sex distributions were similar in the two groups, while the BMls were different; non-survivors included a higher percentage of underweight patients (BMI <18.5 kg/ $\left.\mathrm{m}^{2}\right)(37 \%$ vs. $12.8 \%, \mathrm{p}<0.0001)$, while survivors had a higher percentage of overweight patients (BMI $>25$ $\left.\mathrm{kg} / \mathrm{m}^{2}\right)(27 \%$ vs. $9.2 \%, \mathrm{p}<0.001)$. Practically, all the patients were unaware of their HIV infection status on admission to the RICU; fewer than $4 \%$ were receiving ARV treatment and antimicrobial prophylaxis on admission. Patients in both the groups were severely immunosuppressed, with CD4+ cell counts of 23 (39) (median, interquartile range) and 19 (59) (median, interquartile range) among survivors and non-survivors, respectively. The viral loads were not different between the two groups (5.36 [1.32] vs. 5.59 [1.13] $\log _{10}$ copies $/ \mathrm{ml}$ ). The main reason for admission in both the groups was severe hypoxemic respiratory failure (SHRF) secondary to PJP, requiring invasive ventilation. The number of days of mechanical ventilation, RICU stay, and inhospital stay was not different between the two groups. RICU and inhospital mortality rates were $25.2 \%$ and $26.7 \%$, respectively.

\section{Model development and performance}

A binomial logistic regression was performed to ascertain the effects of underweight, albumin, norepinephrine administration days, and time to RICU admission on the rate of inhospital mortality (Table 2). The linearity of the continuous variables with respect to the logit of the dependent variable was assessed using the Box-Tidwell procedure ${ }^{25}$. A Bonferroni correction was applied using all terms in the model, resulting in statistical significance being accepted when $p<0.00625$. Based on this assessment, all continuous independent variables were found to be linearly related to the logit of the dependent variable. The logistic regression model was statistically significant, $\chi^{2}$ $(4)=54.8, p<0.0005$. The model explained $33.0 \%$ (Nagelkerke $\mathrm{R}^{2}$ ) of the variance in mortality and correctly classified $78.0 \%$ of cases. The area under the 
Table 1. Demographic and clinical characteristics of patients

\begin{tabular}{|c|c|c|c|}
\hline Characteristics & $\begin{array}{l}\text { Survivors } \\
(\mathrm{n}=148)\end{array}$ & $\begin{array}{l}\text { Non-survivors } \\
\quad(n=54)\end{array}$ & $p$ value \\
\hline Age (years) & $33(27 ; 41)$ & $34(29 ; 41)$ & NS \\
\hline Male (\%) & 89 & 92 & NS \\
\hline BMI (\%) & $23.7(20.1 ; 26.3)$ & $20.2(17 ; 23.4)$ & 0.001 \\
\hline Normal (18.5-24.9) & 54 & 51.8 & NS \\
\hline Underweight $(<18.5)$ & 12.8 & 37 & 0.0001 \\
\hline Overweight (25-29.9) & 27 & 9.2 & 0.007 \\
\hline Obesity 1 (30-34.9) & 4.7 & 1.8 & NS \\
\hline Obesity $2(35-39.9)$ & 1.3 & 0 & NS \\
\hline \multicolumn{4}{|l|}{ Education } \\
\hline Primary (\%) & 36.6 & 31.3 & NS \\
\hline Secondary school (\%) & 28.6 & 31.3 & NS \\
\hline High school (\%) & 14 & 9.8 & NS \\
\hline University (\%) & 20.6 & 27.4 & NS \\
\hline \multicolumn{4}{|l|}{ Sexual preference } \\
\hline Heterosexual (\%) & 39.3 & 49 & NS \\
\hline Homosexual (\%) & 56 & 41 & NS \\
\hline Bisexual (\%) & 2.6 & 3.9 & NS \\
\hline Transgender (\%) & 0.6 & 5.8 & NS \\
\hline Condom use (\%) & 17.3 & 7.8 & NS \\
\hline Days since HIV infection diagnosis & $2.0(0 ; 30)$ & $7(0 ; 159)$ & 0.005 \\
\hline $\begin{array}{l}\text { Use of ARV before admission to RICU } \\
(\%)\end{array}$ & 16 & 29 & NS \\
\hline $\begin{array}{l}\text { Use of ARV at the time of admission } \\
\text { to RICU (\%) }\end{array}$ & 3.3 & 3.9 & NS \\
\hline Antimicrobial prophylaxis* (\%) & 3.3 & 3.9 & NS \\
\hline CD4+ count (cells $\mu^{-1}$ ) & $23(11 ; 50)$ & $19(8 ; 65)$ & NS \\
\hline HIV viral load, $\log _{10}\left(\right.$ copies $\mathrm{ml}^{-1}$ ) & $5.36(4.63 ; 5.95)$ & $5.59(4.87 ; 5.93)$ & NS \\
\hline SOFA score & $4(3 ; 8)$ & $8(7 ; 11)$ & 0.001 \\
\hline Albumin & $2.1(1.87 ; 2.5)$ & $1.7(1.17 ; 2.13)$ & 0.001 \\
\hline $\mathrm{DHL}$ & $409(322 ; 518)$ & $427(276 ; 575)$ & NS \\
\hline Norepinephrine days & $5(3 ; 9)$ & $10(6 ; 16)$ & 0.001 \\
\hline Time admission-RICU (days) & $1(0 ; 2)$ & $3(1 ; 8)$ & 0.001 \\
\hline Length of ventilation (days) & $8(5 ; 13)$ & $11(8 ; 18)$ & NS \\
\hline RICU stay (days) & $9(6 ; 14)$ & $13(8 ; 21)$ & NS \\
\hline Hospital stay (days) & $20(14 ; 29)$ & $19(10 ; 30)$ & NS \\
\hline RICU mortality & - & 25.3 & \\
\hline Hospital mortality & - & 26.7 & \\
\hline
\end{tabular}

*Trimethoprim-Sulfamethoxazole. Median ( $25^{\text {th }}$ and $75^{\text {th }}$ percentiles), NS: Non-significant ( $>0.05$ ). BMI: Body mass index (weight in kilograms divided by the square of height in meters), RICU: Respiratory intensive care units, SOFA: Sequential organ failure assessment, HIV: Human immunodeficiency virus, ARV: Antiretroviral. 
Table 2. Multivariate model of predictors of inhospital mortality in patients with human immunodeficiency virus infection and Pneumocystis jiroveci pneumonia

\begin{tabular}{lcccc}
\hline Characteristic & $\beta$ & SE & $\begin{array}{c}\text { OR } \\
(95 \% \mathrm{Cl})\end{array}$ & $\begin{array}{c}\text { Bootstrap bias } \\
(95 \% \mathrm{Cl})\end{array}$ \\
\hline Underweight (BMI <18.5) & 1.197 & 0.431 & $3.31(1.42-7.70)$ & $0.019(0.299-2.208)$ \\
Albumin <1.5 g/dl & 1.066 & 0.447 & $2.90(1.21-6.97)$ & $0.061(0.109-2.146)$ \\
Norepinephrine (days) & 0.108 & 0.029 & $1.11(1.05-1.18)$ & $0.004(0.067-0.158)$ \\
Time to admission to RICU (days) & 0.194 & 0.056 & $1.21(1.08-1.35)$ & $0.014(.088-0.370)$ \\
Constant & -2.983 & 0.406 & & $-0.102(-3.96-2.34)$ \\
\hline
\end{tabular}

BMI: Body mass index, RICU: Respiratory intensive care units, SE: Standard error, Cl: Confidence interval, OR: Odds ratio.

ROC curve was 0.809 ( $95 \% \mathrm{Cl}, 0.721-0.890)$, which is an excellent level of discrimination according to Hosmer et al. ${ }^{26}$.

\section{Mortality score performance}

The score for each of the predictive variables is shown in Table 3. The adjusted logistic regression model for the association between the mortality risk in hospital (p) and the score is given as follows:

$$
\operatorname{Logit}(p)=-5.436+0.481 \text { (score points) }
$$

With a predicted mortality risk estimated by the following equation

Predicted inhospital mortality

$$
\begin{gathered}
=\operatorname{Exp}(-5.436+0.481[\text { score points] } / 1+\exp \\
(-5.436+0.481[\text { score points] })
\end{gathered}
$$

The overall performance of the score (NagelkerkeR ${ }^{2}$ ) was 0.467; the discrimination (area under the ROC curve) was 0.869 (95\% Cl: 0.821-0.917) (Fig. 2) and calibration intercept $(\alpha)$ and slope $(\beta)$ were 0.03 and 0.99 , respectively. The sensitivity was $47.2 \%$, the specificity was $84.6 \%$, the positive predictive value was $89.2 \%$, and the negative predictive value was $82.6 \%$.

Based on the obtained scores, the population was initially divided into quartiles to obtain risk groups. Since there was no significant difference between Groups 2 and 3, these were merged into an intermediate risk group. Finally, the three risk groups were integrated: low risk with a score of 0-6 and a
Table 3. Scores assigned to the different levels of the mortality predictor variables

\begin{tabular}{ll}
\hline Predictor & Points \\
\hline BMI $\left(\mathrm{g} / \mathrm{m}^{2}\right)$ & 0 \\
$18.5-25$ & 2 \\
$18.5-17.5$ & 5 \\
$17.4-16.5$ & 7 \\
$<16.5$ & \\
Albumin (g/dl) & \\
$4.5-3.5$ & 0 \\
$3.4-2.5$ & 1 \\
$2.4-1.5$ & 3 \\
$1.4-0.5$ & 5 \\
Time to RICU admission (days) & \\
$0-3$ & 0 \\
$4-7$ & 1 \\
$8-11$ & 2 \\
$12-15$ & 3 \\
$>16$ & 4 \\
Days with norepinephrine & \\
$1-5$ & 4 \\
$6-10$ & \\
$11-15$ & \\
$>20$ & \\
\hline
\end{tabular}

BMI: Body mass index, RICU: Respiratory intensive care units.

probability of mortality of $2.6 \%$, intermediate risk with a score of 7-12 and a probability of mortality of $36.1 \%$, and high risk with a score of $\geq 13$ and a probability of mortality of $63.3 \%$. Log-rank pairwise comparisons were performed to determine which 
Figure 2. Receiving operating characteristic curve for the derived score. Area under the curve $=0.869$ ( $95 \%$ confidence interval: 0.821-0.917).

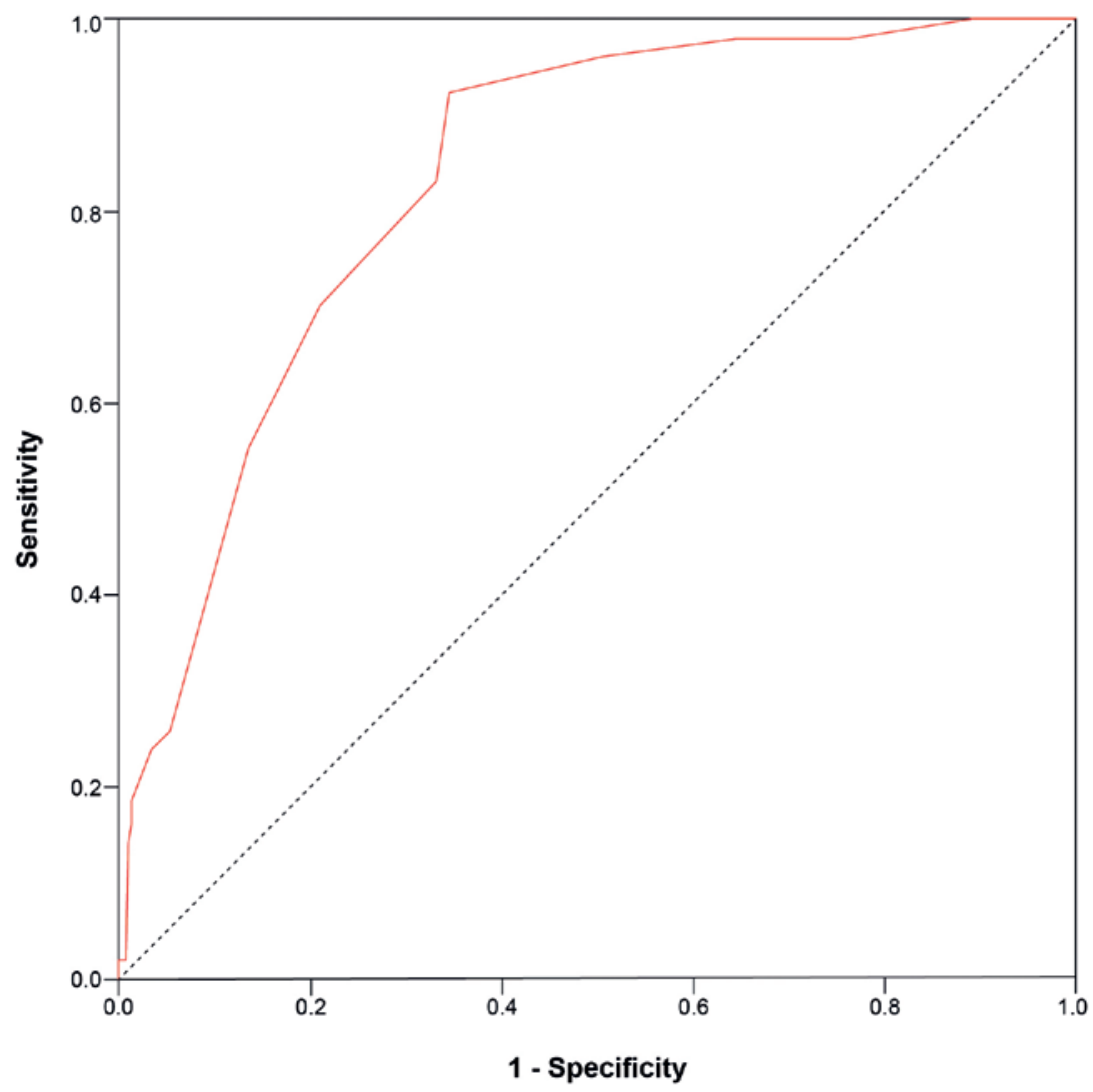

Figure 3. Kaplan-Meier survival curves show the distribution of survival among different risk groups. There was a statistically significant difference in survival distribution between groups as determined by log-rank pairwise comparisons.

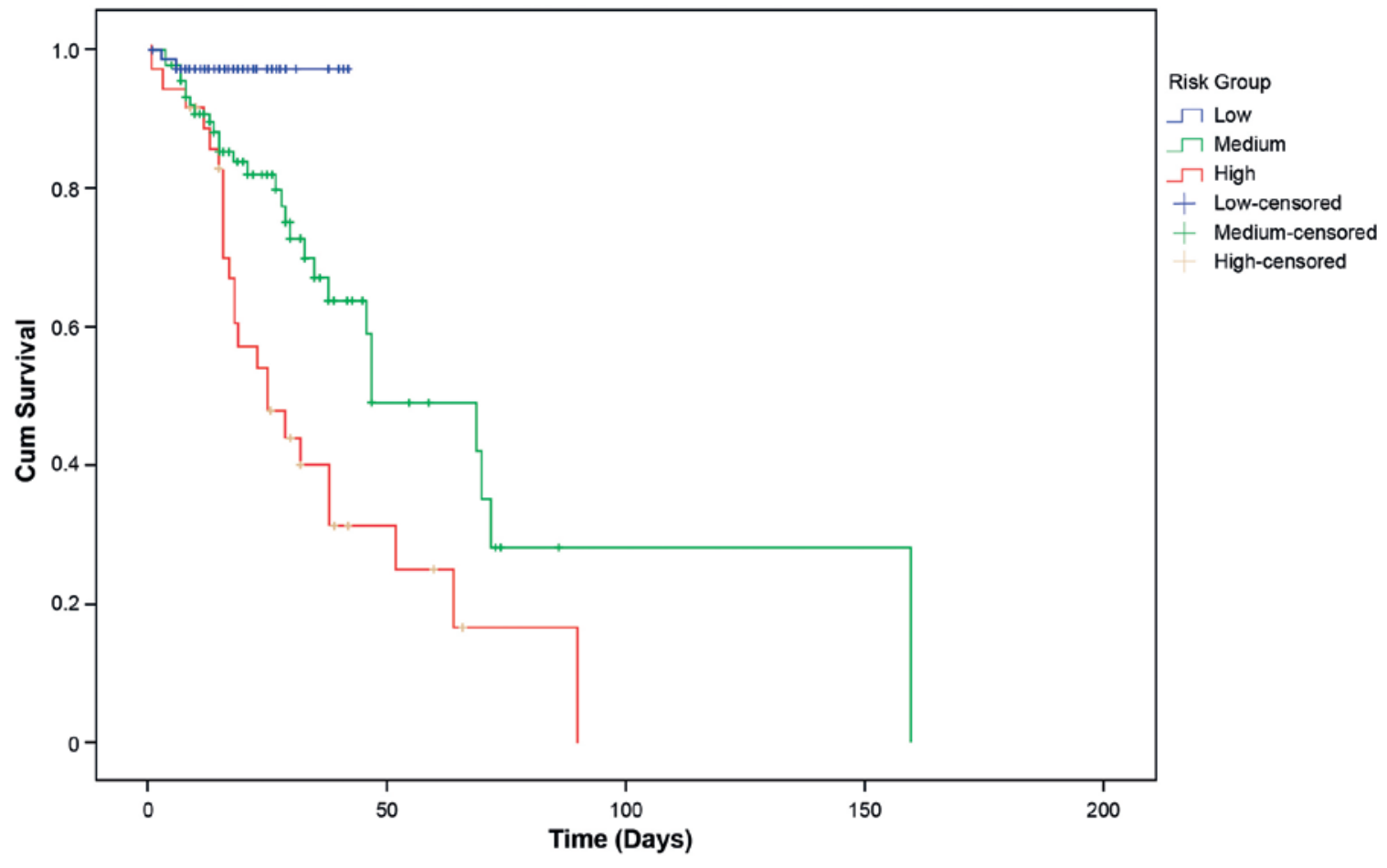


intervention groups had different survival distributions. A Bonferroni correction was made with statistical significance accepted at $p<0.0167$. There was a statistically significant difference in survival distributions for the risk groups: low versus intermediate $(p=0.003)$, low versus high $(p<0.0005)$, and intermediate versus high $(p=0.003)$ (Fig. 3 ).

\section{DISCUSSION}

In this study, we described the clinical characteristics and outcome in a cohort of HIV/AIDS patients with $P$. jiroveci pneumonia and severe acute hypoxemic respiratory failure and developed a predictive scoring system to estimate the risk of mortality in these patients. Our results show that three factors that are easily obtainable on the patient's admission to the ICU (BMI, serum albumin, and time interval between admission to the emergency room and admission to the RICU) predict inhospital mortality. The duration of vasopressor support was also significantly predictive of mortality. Having a tool to predict the probability of survival is important for patients, families, and hospital personnel before selecting the appropriate treatment given the ethical and economic implications that these decisions entail. As such, the scoring system developed in our unit may guide these decisions.

Several markers of malnutrition have been associated with excess mortality in severely ill patients, including low BMl or body weight ${ }^{27,28}$. A low BMI has also been associated with a prolonged hospital stay, an increased risk of nosocomial infection, and a greater number of days of mechanical ventilation ${ }^{29,30}$. In our population of patients with HIV infection and SAHRF, a low BMI $\left(<18.5 \mathrm{~kg} / \mathrm{m}^{2}\right)$ showed a significant independent association with inhospital mortality, which is consistent with previous results obtained in critically ill HIV-infected and non-HIV-infected patients ${ }^{27-31}$. These data highlight nutritional status as an important factor for survival in critically ill HIVinfected patients. HIV patients with high viral loads have been shown to have a hypermetabolic state mediated by elevated levels of inflammatory cytokines with increased basal energy expenditure and accelerated proteolysis ${ }^{32}$. The median viral loads of our patients, survivors and non-survivors, were 5.36 and $5.59 \log _{10}$ copies/ml, respectively. In this context, early detection of HIV infection is important, as studies show that delaying ARV initiation increases the risk of opportunistic infections that contribute significantly to HIV-associated wasting syndrome ${ }^{28,33}$. Therefore, it is of concern that, in $94 \%$ of our patients, the diagnosis of AIDS and PJP was made at the time of admission, which was reflected in high viral loads and low CD4 cell counts. Early detection requires strengthening and restructuring primary care medical services in the country, a task for which the coordinated participation of the government and private investment is indispensable.

Hypoalbuminemia is reportedly an independent risk factor for mortality in practically all studies performed in patients with HIV infection and has also been reported as an independent predictor of non-AIDS complications and long-term mortality in patients living with $\mathrm{HIV}^{34}$. Albumin has antioxidant activity and can, therefore, protect cells from oxidative stress during sepsis $^{35}$. It is also a biomarker of inflammation; albumin levels decrease during acute and chronic inflammatory processes due to the inhibition of its synthesis in the liver by cytokines ${ }^{36}$. Our results showed a strong association between low serum albumin levels and mortality, which may indicate greater chronicity or a more intense inflammatory response in patients with lower albumin levels and higher mortality.

Septic shock and the use of vasopressors are predictors of mortality in patients with PJP and HIV infection $^{37}$. We found that the number of days with vasopressor support was an independent risk factor for mortality. While unknown at the time of admission, we included this factor due to its weight as a mortality predictor, and because it may serve to reevaluate expectations of survival throughout a patient's course.

At the onset of the AIDS epidemic, the survival rate of patients with PJP-associated SHRF who required intubation and mechanical ventilation was no higher than $15 \%$, making it difficult to justify their admission to an $\mathrm{ICU}^{4}$. However, their survival rates have since improved substantially ${ }^{38}$; among our patients, the survival rate was $>70 \%$ on discharge from the hospital. Similar results have been reported by other researchers ${ }^{3,8,9}$. Since none of our patients were receiving ARV therapy or PJP prophylaxis at the time of admission, survival times similar to those reported in 
the pre-ARV era would be expected. The improvement in survival, therefore, appears to be independent of ARV therapy and most likely reflects advances in intensive care and respiratory management ${ }^{39}$. Rapid admission to the ICU, the application of lung-protective ventilation, and, in selected cases, advanced measures can be important to improve the survival of these patients.

Efforts to develop scoring systems predictive of prognosis in patients with HIV infection and PJP started at the beginning of the AIDS epidemic. Montaner et al. ${ }^{5}$ retrospectively evaluated various scoring systems to predict mortality in 56 patients with SHRF due to PJP who required intensive care between 1985 and 1991; their mortality rates were 65\% overall and $72 \%$ in those who required mechanical ventilation. On multivariate analysis, only the "multiple system organ failure" score predicted mortality; its performance was enhanced when the serum lactate dehydrogenase (LDH) level was included in the model. The vasopressor requirement was also a significant predictor of mortality in this cohort. Speich et al. ${ }^{6}$ proposed a PJP severity scoring system based on the following parameters: LDH level, $P(A-a) O^{2}$, and the percentage of neutrophils in the bronchial lavage fluid obtained in the first 24-48 $\mathrm{h}$ after admission. Only 4 of the 94 patients required mechanical ventilation, and the 14 -day mortality rate was $7 \%$. Despite the importance of these first studies in developing a predictive prognostic model, they have important limitations in current clinical applications due to changes in populations and treatment methods over time ${ }^{14}$.

Our developed model and scoring system for predicting mortality showed good discriminatory capacities, with an area under the ROC curve of 0.869 (i.e., if two patients in this population were randomly selected, the probability that the model correctly identified the non-survivor would be $86.9 \%$ ). Calibration analysis showed an excellent match between the predicted and observed probabilities ( $\alpha=0.03$ and $\beta=0.99$ ). In addition, the model was validated internally and did not show a significant bias. Hence, the model and the scoring system derived from it can be used as tools to help clinicians in decision-making and communicate expectations regarding the probability of patient death with family members. In addition, the model can be useful for the quality control of ICU medical care because important deviations from the mortality predicted in different strata ought to lead to revising management protocols.

However, it is important to discuss the limitations of the study so that clinicians are fully informed of any drawbacks of this statistical tool. Although our model showed good calibration and discrimination and was internally validated, external validation remains necessary for a proper evaluation of our model's generalization. In this context, our model requires recalibrating in ICUs that wish to adopt it.

In summary, we developed a model and prognostic scoring system using variables that are easy to obtain on admission to the ICU in HIV/AIDS patients with severe acute hypoxemic respiratory failure caused by PJP. The internally validated scoring system, which showed good calibration and discrimination, provides a potentially useful tool to assist clinicians to estimate the inhospital mortality risk in a specific HIV/ AIDS patient population. However, more studies are required to validate the application of our score, especially in low- and middle-income countries, where PJP continues to be a significant burden for health services.

\section{REFERENCES}

1. Joint United Nations Programme on HIV and AIDS (UNAIDS). Mexico. Available From: http://www.unaids.org/es/regionscountries/countries/mexico. [Last accessed on 2018 Oct 06].

2. Vincent B, Timsit JF, Auburtin M, et al. Characteristics and outcomes of HIV-infected patients in the ICU: impact of the highly active antiretroviral treatment era. Intensive Care Med. 2004; 30:859-66.

3. Powell J, Davis JL, Morris AM, et al. Survival for patients with human immunodeficiency virus admitted to the intensive care unit continues to improve in the current era of highly active antiretroviral therapy. Chest. 2009;135:11-17.

4. Wachter RM, Luce JM, Turner J, Volberding P, Hopewell PC. Intensive care of patients with the acquired immunodeficiency syndrome. Outcome and changing patterns of utilization. Am Rev Respir Dis. 1986;134:891-6.

5. Montaner JS, Hawley PH, Ronco JJ, et al. Multisystem organ failure predicts mortality of ICU patients with acute respiratory failure secondary to AIDS-related PCP. Chest. 1992;102:18238.

6. Speich R, Opravil M, Weber R, et al. Prospective evaluation of a prognostic score for Pneumocystis carinii pneumonia in HIV-infected patients. Chest. 1992;102:1045-8.

7. Huang L, Quartin A, Jones D, Havlir DV. Intensive care of patients with HIV infection. N Engl J Med. 2006;355:173-81.

8. Coquet I, Pavie J, Palmer P, et al. Survival trends in critically ill HIV-infected patients in the highly active antiretroviral therapy era. Crit Care. 2010;14:R107.

9. Fisk DT, Meshnick S, Kazanjian PH. Pneumocystis carinii pneumonia in patients in the developing world who have acquired immunodeficiency syndrome. Clin Infect Dis. 2003;36:70-8.

10. Billings JD, Davey DL, Konda KA, et al. Factors associated with previously undiagnosed human immunodeficiency virus infec- 
tion in a population of men who have sex with men and maleto-female transgender women in Lima, Peru. Medicine (Baltimore). 2016;95:e5147.

11. Collins GS, Reitsma JB, Altman DG, Moons KG. Transparent reporting of a multivariable prediction model for individual prognosis or diagnosis (TRIPOD): the TRIPOD statement. BMJ 2015; 350:g7594.

12. Mehta RL, Kellum JA, Shah SV, et al. Acute kidney injury network: report of an initiative to improve outcomes in acute kidney injury. Crit Care. 2007;11:R31.

13. Vincent JL, Moreno R, Takala J, et al. The SOFA (Sepsis-related organ failure assessment) score to describe organ dysfunction/ failure. On behalf of the working group on sepsis-related problems of the European society of intensive care medicine. Intensive Care Med. 1996;22:707-10.

14. Ferguson ND, Fan E, Camporota $L$, et al. The berlin definition of ARDS: an expanded rationale, justification, and supplementary material. Intensive Care Med. 2012;38:1573-82.

15. Marshall A, Altman DG, Holder RL, Royston P. Combining estimates of interest in prognostic modelling studies after multiple imputation: current practice and guidelines. BMC Med Res Methodol. 2009;9:57

16. Vesin A, Azoulay E, Ruckly S, et al. Reporting and handling missing values in clinical studies in intensive care units. Intensive Care Med. 2013;39:1396-404.

17. Collett D. Modelling Binary Data. $2^{\text {nd }}$ ed. New York: Chapman and Hall/CRC; 2003.

18. Sullivan LM, Massaro JM, D'Agostino RB Sr. Presentation of multivariate data for clinical use: the Framingham study risk score functions. Stat Med. 2004;23:1631-60.

19. Miller ME, Langefeld CD, Tierney WM, Hui SL, McDonald C]. Validation of probabilistic predictions. Med Decis Making. 1993; 13:49-58.

20. Nagelkerke NJ. A note on a general definition of the coefficient of determination. Biometrika 1991;78:691-692.

21. Hanley JA, McNeil BJ. The meaning and use of the area under a receiver operating characteristic (ROC) curve. Radiology. 1982; 143:29-36

22. Van Calster B, Nieboer D, Vergouwe $Y$, et al. A calibration hierarchy for risk models was defined: from Utopia to empirical data. J Clin Epidemiol. 2016;74:167-76.

23. Steyerberg EW, Harrell FE Jr., Borsboom GJ, et al. Internal validation of predictive models: efficiency of some procedures for logistic regression analysis. J Clin Epidemiol. 2001;54:774-81.

24. Peduzzi P, Concato J, Kemper E, Holford TR, Feinstein AR. A simulation study of the number of events per variable in logistic regression analysis. J Clin Epidemiol. 1996;49:1373-9.
25. Box GE, Tidwell PW. Transformation of the independent variables. Technometrics. 1962;4:531-550

26. Hosmer DW Jr., Lemeshow S, Sturdivant RX. Applied Logistic Regression. 3rd ed. Hoboken, NJ: Wiley; 2013.

27. Garrouste-Orgeas M, Troché G, Azoulay E, et al. Body mass index. An additional prognostic factor in ICU patients. Intensive Care Med. 2004;30:437-43.

28. Mangili A, Murman DH, Zampini AM, Wanke CA. Nutrition and HIV infection: review of weight loss and wasting in the era of highly active antiretroviral therapy from the nutrition for healthy living cohort. Clin Infect Dis. 2006;42:836-42.

29. Yatabe T, Yamashita K, Yokoyama M. Lower body mass index is associated with hospital mortality in critically ill Japanese patients. Asia Pac J Clin Nutr. 2016:25:534-7.

30. Pickkers $P$, de Keizer N, Dusseljee J, et al. Body mass index is associated with hospital mortality in critically ill patients: an observational cohort study. Crit Care Med. 2013;41:1878-83.

31. Zampieri FG, Colombari F. A gradient-boosted model analysis of the impact of body mass index on the short-term outcomes of critically ill medical patients. Rev Bras Ter Intensiva. 2015; 27:141-8

32. Melchior JC, Salmon D, Rigaud D, et al. Resting energy expenditure is increased in stable, malnourished HIV-infected patients. Am J Clin Nutr. 1991;53:437-41.

33. Shikuma CM, Zackin R, Sattler F, et al. Changes in weight and lean body mass during highly active antiretroviral therapy. Clin Infect Dis. 2004;39:1223-30.

34. Ronit A Sharma S, Baker JV et al Serum albumin as a prognostic marker for serious non-AIDS endpoints in the strategic timing of antiretroviral treatment (START) study. J Infect Dis. 2018;217:405-12

35. Taverna M, Marie AL, Mira JP, Guidet B. Specific antioxidant properties of human serum albumin. Ann Intensive Care. 2013;3:4

36. Di Napoli $\mathrm{M}$, Behrouz $\mathrm{R}$, Topel $\mathrm{CH}$, et al. Hypoalbuminemia, systemic inflammatory response syndrome, and functional outcome in intracerebral hemorrhage. J Crit Care. 2017;41:247-53.

37. Barbier F, Coquet I, Legriel S, et al. Etiologies and outcome of acute respiratory failure in HIV-infected patients. Intensive Care Med. 2009;35:1678-86.

38. Miller RF, Allen E, Copas A, Singer M, Edwards SG. Improved survival for HIV infected patients with severe Pneumocystis jirovecii pneumonia is independent of highly active antiretroviral therapy. Thorax. 2006;61:716-21.

39. Davis JL, Morris A, Kallet RH, et al. Low tidal volume ventilation is associated with reduced mortality in HIV-infected patients with acute lung injury. Thorax. 2008;63:988-93. 